\title{
Validation of self-report and hospital pill count using unannounced home pill count as meth- ods for determination of adherence to antiretroviral therapy
}

\author{
O.M.S. MINZI* and A.S. NAAZNEEN \\ Muhimbili University of Health and Allied Sciences, School of Pharmacy, P.O. Box 65013, Dar es Salaam, Tanzania
}

\begin{abstract}
Adherence to anti-retroviral therapy (ART) is very crucial for successful treatment outcomes. This study aimed to validate patient-self report (PSR) and hospital based pill count (HoPC) as adherence determination methods by using unannounced home visit pill count (HPC). The study was carried out at Muhimbili National Hospital in Dar es Salaam, Tanzania and 215 patients purposively selected were recruited. On refill day, the remaining pills were counted. They were also asked to report on the number of doses they missed during the past 28 days. They were later visited in their homes without appointment where the remaining pills were counted. Ninety-eight percent and $93 \%$ reported to adhere to ART by PSR method and HoPC, respectively. However, only $58 \%$ of the same study patients were found to be adherent by $\geq 95 \%$ using HPC. In conclusion, PSR and HoPC do not always give reliable adherence data in patients undergoing ART. Therefore, we recommend application of combination methods for adherence measuring in patients starting to include patient self report and hospital based pill count in new patients and complementing them with unannounced home based pill count in experienced patients. Wherever possible, drug plasma concentration measurements should also be established.
\end{abstract}

Key words: anti-retroviral therapy, adherence, self-report, pill count, HIV/AIDS, Tanzania

\section{Introduction}

Human Immunodeficiency Virus (HIV) infection continue to create a public health concern worldwide and more than 38.6 million people were infected by the end of 2005 (UNAIDS, 2006). In response to this, most affected countries, including Tanzania, declared HIV infection as a state of emergency and started providing antiretroviral therapy (ART) early in 2000s. ART suppresses viral load and raises the number of CD4 cells, therefore improving the quality of life of HIV infected patients. ART is a life long therapy and requires stringent adherence to treatment. Studies have shown that for a treatment success, adherence to ART should be $\geq 95 \%$ (Chesney et al., 1999; Read et al., 2003; Garcia et al., 2003). Lack of adherence to ART may lead to therapeutic failure, deterioration of the immune system and/or emergence of medicine-resistant HIV strains which in turn, will lead to escalating costs of care (Munakata et al., 2006).

There are many factors which contribute to poor ART adherence, among them being complexity of ART regimen, drug toxicity, pill burden, forgetting and poor drug supply chain (Tseng, 1998). Different methods for determining adherence to ART, such as pill-count, patient self-report, and biological markers such as CD4 count and viral load count, have been reported (Tseng, 1998; Bentwich, 2005). Currently, hospital based pill count and patient-self reported adherence methods are routinely recommended for determination of adherence rate to ART in care and treatment clinics in Tanzania. However, such methods have been blamed to be subjective, giving an overestimate of adherence in many countries and therefore posing a concern on the reliability of data obtained (Rudd et al., 1990; Waterhouse et al., 1993; Urquhart 1994; Urquhart \& de Klerk, 1998). The objective of this study therefore, was to validate data of ART adherence obtained by self report and hospital based pill count as compared to unannounced home visit pill counts in patients who have been on ART for at least eight months.

\section{Materials and Methods}

\section{Study design, subject selection}

This was a cohort, prospective, non randomized study involving 215 HIV infected patients attending Care and Treatment Clinic (CTC) at Muhimbili National Hospital in Dar es Salaam, Tanzania. Muhimbili National Hospital is the leading and largest referral hospital in the country receiving referred patients from all Dar es Salaam municipal hospitals and other parts of the country. It has the busiest care and treatment clinic in the country with more than 2800 adult HIV patients and 600 children currently on ART. The patients were purposively selected in six separate study episodes. The study was carried out from October 2006 to April, 2007. Only patients whose medical records (CTC2 form) showed a decline in CD4 count and/or progres- 
sion of clinical symptoms were included in the study. In each episode, patients were studied using both pill count, patient self report and unannounced home visit pill count methodologies. In addition, the patient records were viewed retrospectively.

The patients included in this study were those who were using a combination of stavudine (40mg), lamivudine (150 mg) and nevirapine (200 $\mathrm{mg}$ ). The drugs were supplied as one tablet fixed dose combination containing the 3 drugs in the mentioned proportion. The patients were instructed to swallow a single tablet of such a combination every after 12 hours. On refill day, they were always dispensed an intact tin containing 60 tablets. The patients were also advised to come back for refill at least 3 days before the dispensed doses exhaustion. During recruitments, a total of 215 patients were enrolled starting with 33,42 , $40,35,32$ and 33 in six separate recruiting episodes, respectively. Clinical records of patients were viewed for $\mathrm{CD} 4$ count data and clinical symptoms from CTC2 form. The study was conducted at the HIV/AIDS care and treatment clinic at Muhimbili National Hospital. This clinic provides free antiretroviral treatment and dispenses ARVs for refill monthly.

\section{Hospital pill count, patient self-report and un-an- nounced home based pill report}

Enrolled patients were requested to bring the remaining pills in their original containers of 60 pills on the next scheduled visit and the pills were counted. The number of pills remaining unused was related to the date of the previous refill obtained from patient card (CTC1 form). Patients were asked to recall the number of ARV doses they missed for the past 28 days. They were also asked if they took their medicines on time as required. On each patient identification (ID) card details that were used to trace the patients in their domicile: patient ID number, name, date of birth, sex, telephone, district, division, ward, street/village, name of the community leader, address and telephone of the patient assistant. In addition the patients were asked to provide their physical addresses to counsellors on the day they attended the CTC for ARV refill. Three weeks after refill, the home-based care providers paid unannounced visits to their homes. The health care providers counted the remaining pills and calculated how many pills were supposed to have been swallowed by the patient in relation to the date of previous refill which was obtained from patient ID card. Adherence was calculated by determining the number of pills used against the expected number of pills to have been used by the patient.

\section{Documentation of the clinical performance of pa- tients}

Baseline information on immunological conditions of patients was obtained from patient records kept at the clinic in CTC2 form in which their CD4 count before and after every six months after initiation of ART was recorded.

\section{Data analysis}

Adherence by patient self report was estimated by calculating for each patient the total number of doses reported to have been taken over the specified period of time (28 days) and dividing by the total number of doses dispensed for that period. Adherence by both hospital and unannounced home pill count was calculated based on the ratio of the actual number of pills taken and the expected number of pills which were supposed to have been taken from the day of last refill until the day of pill count. Patients were judged to be adherent if they met the criteria of $\geq$ $95 \%$ adherence rate. The mean percentage of adherent patients obtained from hospital based pill count and self report methods were compared with that obtained from unannounced home pill count using paired student $t$-test. The difference was regarded as statistically significant if there was a difference of $\geq$ $25 \%$ between the comparisons.

\section{Ethical consideration}

Participants were not informed on the purpose for the study so as to avoid modification of their drug intake behaviour. Permission to conduct the study at Muhimbili National Hospital was obtained from the administration of the Out-Patient Unit of the Department of Internal Medicine.

\section{Results}

In total, 215 AIDS patients participated in the study and were recruited in six separate episodes in the order of $33,42,40,35,32,33$ patients. Among them $28 \%$ were males aged between 30 and 49 years (median $38 \pm 2$ years) and $72 \%$ were females of ages ranging from 20 to 45 years (median $30 \pm 4$ years). Looking into patient self report taken from recruitment episode 6 , it can be seen that almost all patients reported to be adherent (range of adherence 96-100\%). The same applied with hospital based pill count (Table 1).

The proportions of patients who were found to be adherent by $\geq 95 \%$ using all the 3 methods are summarised in Table 2 . The majority of the subjects 
Table 1: Patient cases and the trend of CD4 counts overtime obtained from patient records in episode 6

\begin{tabular}{lcccc}
\hline $\begin{array}{l}\text { Patient } \\
\text { number }\end{array}$ & $\begin{array}{l}\text { Adherence in \% } \\
\text { obtained by PSR }\end{array}$ & $\begin{array}{c}\text { Adherence in \% } \\
\text { obtained by HoPC }\end{array}$ & $\begin{array}{c}\text { Adherence in \% } \\
\text { obtained by HPC }\end{array}$ & $\begin{array}{l}\text { CD4 count from } \\
\text { clinical records }\end{array}$ \\
\hline 1 & 100 & 94 & 70 & $168-93$ \\
2 & 96 & 93 & 76 & $165-103$ \\
3 & 97 & 92 & 67 & $200-116$ \\
4 & 100 & 100 & 69 & $198-99$ \\
5 & 98 & 90 & 70 & $200-177$ \\
6 & 100 & 100 & 100 & $124-94$ \\
7 & 100 & 110 & 100 & $173-122-105-91$ \\
8 & 100 & 103 & 89 & $129-178-103$ \\
\hline
\end{tabular}

$\mathrm{PSR}=$ Patient self-report, $\mathrm{HoPC}=$ Hospital pill count $\mathrm{HPC}=$ Unannounced home visit pill count

Table 2: Mean percent of patients found to be adherent by $\geq 95 \%$ obtained by using all the 3 adherence assessment methods from 6 episodes

\begin{tabular}{|c|c|c|c|}
\hline Episode & $\begin{array}{l}\text { Mean } \% \pm \text { SD of } \\
\text { patients adherent by } \\
\geq 95 \% \text { obtained by PSR }\end{array}$ & $\begin{array}{l}\text { Mean } \% \pm \text { SD of patients } \\
\text { adherent by } \geq 95 \% \\
\text { obtained by HoPC }\end{array}$ & $\begin{array}{l}\text { Mean } \% \pm \text { SD of patients } \\
\text { adherent by } \geq 95 \% \\
\text { obtained by HPC }\end{array}$ \\
\hline $1(n=33)$ & $99 \pm 3$ & $98 \pm 4$ & $45 \pm 6$ \\
\hline $2(n=42)$ & $100 \pm 5$ & $92 \pm 5$ & $65 \pm 5$ \\
\hline $3(n=40)$ & $97 \pm 2$ & $97 \pm 4$ & $64 \pm 4$ \\
\hline $4(n=35)$ & $99 \pm 4$ & $93 \pm 4$ & $43 \pm 5$ \\
\hline $5(n=32)$ & $98 \pm 6$ & $92 \pm 3$ & $50 \pm 7$ \\
\hline $6(n=33)$ & $97 \pm 4$ & $89 \pm 8$ & $64 \pm 5$ \\
\hline Mean $(n=215)$ & $98 \pm 2$ & $93 \pm 5$ & $55 \pm 4$ \\
\hline
\end{tabular}

(98\% and $93 \%$ ) were found to be adherent by $\geq 95 \%$ by patient self-report and hospital based pill count methods. However, data obtained from un-announced home-based pill count indicate a much lower proportion $(55 \%)$ in the same patients. In both cases, self report showed the highest number of the calculated patients adhering well to ART.

By using unannounced home pill count, we found out that, there was an average of $10 \pm 1$ (median $6 \pm 2$ ) of unused pills in relation to the number of days which the patient was expected to have used the drugs.

\section{Discussion}

This study has revealed that both patient self-report and hospital pill count do not give the true picture of adherence in ART patients. By using PSR, there is always an overestimate of adherence as observed in our study in which there was an overestimate of adherence by $35-40 \%$ compared to home pill count. This means that patients do not always report the truth to health care providers. There are many factors which have been reported to undermine the reliability of self-report method including recall bias, illiteracy and patient's fear to physicians to report the truth
(Rudd et al., 1990, Waterhouse et al., 1993, Tseng, 1998,). Among factors related to treatment failure, non-adherence is a primary factor influencing ART response (Mannheimer et al., 2002, Munakata et al., 2006). Poor adherence has got consequences on the therapeutic life span of ARVs. First line ARV treatment combinations in Tanzania require a combination of 2 nucleoside reverse transcriptase inhibitors (NRTIs) and one non-nucleoside reverse transcriptase inhibitor (NNRTI). The NNRTIs currently recommended in the country are nevirapine and efavirenz. A cross resistance between the two drugs has been reported whence, a single point mutation to nevirapine would automatically lead to resistance against the later (Delaugerre et al., 2001).

Analyzing the data obtained from unannounced home visit pill count, there was a dramatic fall of adherence in those patient found to be adherent by using hospital pill count and self report method. A decline of CD4 count in patients 6 and 7 who were found to have good adherence by both methods could be probably a case where already there was resistance against the drug combination used. The treatment guidelines demand switching to second line drugs whenever there is treatment failure with the first line ART after ruling 
out non-adherence. By looking into the trend of CD4 fall observed in this study, one could draw a false conclusion that already the ARVs used had no treatment efficacy, whereas actually the problem is pseudo high adherence obtained by self-report and hospital pill count methods. However, by analyzing the data obtained by using unannounced home pill count in the same study patients, only $55 \%$ of the patients are found to be adherent indicating an overestimate of more than $35 \%$ in both cases.

Currently, most ART facilities in the country use patient self-report and hospital pill count as the methods of choice for measuring adherence to ART. Our study has shown that neither patient- reported adherence nor hospital pill count give true picture of adherence to ART but an overestimate of adherence as opposed to the data obtained from unannounced home pill count. Unannounced home visit pill count could therefore be an alternative method to patient self-report in some cases. However, the method has setback due to the fact that the method involves incurring expenses through visiting patients at home which could be an issue in most developing countries where the HIV/AIDS pandemic has seriously hit. Moreover, the visits must be done without appointment which may add to the cost as patients are not always available at their homes.

We could not determine in this study the reasons for such a big difference in adherence rates obtained by self-report and unannounced home based pill count in the same study patients. Recently, Bentwich (2005) reported that the habit of some patients dumping some ARV pills before attending a refill, thus creating the sense of pseudo adherence.

Self-report and hospital based pill count methods are not costly and are feasible for routine applications. Such methods should be reserved for providing preliminary information on ART adherence during early stages of ART in patients. Self report and hospital pill count in ART could be useful in new ART patients but as the patient becomes more experienced with the treatment, he/she loses the fear of dying from AIDS especially after noticing a clinical improvement and therefore may deliberately skip some doses and invent mechanisms of lying to the care providers. An unannounced home visit does not provide opportunities for a patient to temper with the number of remaining pills.

Recently, antiretroviral plasma concentrations were reported to be used as alternative reliable adherence markers in patients undergoing ART (Kastrissios et al., 1998; Yasuda et al., 2004). We have verified in this study that neither hospital based pill count nor patient self report alone do always give reliable information on adherence from patients undergoing ART. The ART programmes should adopt a set of adherence determination methods which complement each other and could consider establishing few centres for determination of ARV plasma concentrations where some samples from patients showing poor treatment outcomes could be tested.

\section{Acknowledgements}

We thank the HIV/AIDS patients for voluntary participation in the study. We also thank the Management of the Muhimbili National Hospital for their support. We are grateful to Sisters Rosalia Luhoyo and Happiness Lyimo for their assistance in data collection. This study was funded by the Higher Education Loan Board of Tanzania.

Received January 27, 2008

Revised 21 March 2008

Accepted 22 March 2008

\section{References}

Bentwich, Z. (2005) CD4 measurements in patients with HIV: are they feasible for poor settings? PLoS Medicine 2, 595-596.

Chesney, M.A., Ickovics, J., Hecht, F., Sikipa, G. \& Rabkin, J. (1999) Adherence: a necessity for successful HIV combination therapy. AIDS 13, 271-278.

Delaugerre, C., Rohban., R., Simon, A., Mouroux, M., Tricot, C., Agher, R., Huraux, J.M., Katlama, C. \& Calvez, V. (2001) Resistance profile and cross-resistance of HIV-1 among patients failing a non-nucleoside reverse transcriptase inhibitor-containing regimen. Journal of Medical Virology 65, 445- 448.

Garcia, R., Schooley, R.T. \& Badaró, R. (2003) An adherence trilogy is essential for long-term HAART success. Brazilian Journal of Infectious Diseases 7, 307-314.

Kastrissios, H., Suarez, J.R., Hammer, S., Katzenstein, D. \& Blaschke, T.F. (1998) The extent of nonadherence in a large AIDS clinical trial using plasma dideoxynucleoside concentrations as a marker. AIDS 12, 2305-2311.

Mannheimer, S., Frieland, G., Matts, J., Child, C. \& Chesney, M. (2002) The consistency of adherence to antiretroviral therapy predicts biologic 
outcomes for human immunodeficiency virusinfected persons in clinical trials. Clinical Infectious Diseases 34, 1115-1121.

Munakata, J., Benner, J.S.,Becker, S.,Dezii, C.M., Hazard, E.H. \& Tierce, J.C. (2006) Clinical and economic outcomes of non-adherence to highly active antiretroviral therapy in patients with human immunodeficiency virus. Medical Care 44, 893-899.

Read, T., Mijch, A. \& Fairley, C.K. (2003) Adherence to antiretroviral therapy: are we doing enough? International Medicine Journal 33, 254-256.

Rudd, P., Ahmed, S. \& Zachary, V. (1990) Improved compliance measures: application in an ambulatory hypertensive drug trial. Clinical Pharmacokinetics and Therapeutics 48, 676-685.

Tseng, A.L. (1998) Compliance issues in the treatment of HIV infection. American Journal of Health-System Pharmacy 55, 1817-1824.

UNAIDS (2006) Report on the Global AIDS epidemic. United Nations AIDS. (http://www.unaids.
org/en/HIV data/2006GlobalReport/default. asp).

Urquhart, J. \& de Klerk, E. (1998) Contending paradigms for the interpretation of data on patient compliance with therapeutic drug regimens. Statistical Medicine 17, 251-267.

Urquhart, J. (1994) The role of patient compliance in clinical pharmacokinetics. A review of recent research. Clinical Pharmacokinetics 27, 202-215.

Waterhouse, D.M., Calzone, K.A. \& Mele, C. (1993) Adherence to oral tamoxifen: a comparison of patient self report, pill counts and microelectronic monitoring. Journal of Clinical Oncology 11, 189-1197.

Yasuda, J.,M., Miller, C., Currier, J.S., Forthal, D.N., Kemper, C.A., Beall, G.N., Tilles, J.G., Capparelli, E.V., McCutchan, J.A. \& Haubrich, R.H. California Collaborative Treatment Group (2004) The correlation between plasma concentrations of protease inhibitors, medication adherence and virological outcome in HIV-infected patients. Antiretroviral Therapy 9, 753-761. 